\title{
VERDICT prostate parameter estimation with AMICO
}

\author{
Elisenda Bonet-Carne ${ }^{12} \bowtie$, Alessandro Daducci ${ }^{34}$, Edward Johnston ${ }^{2}$, Joseph \\ Jacobs $^{1}$, Alex Freeman ${ }^{5}$, David Atkinson ${ }^{2}$, David J. Hawkes ${ }^{6}$, Shonit \\ Punwani $^{2}$, Daniel C. Alexander ${ }^{1}$, and Eleftheria Panagiotaki ${ }^{1}$ \\ 1 UCL Centre of Medical Imaging Computing, Department of Computer Science, \\ London, UK \\ 2 UCL Centre for Medical Imaging, Division of Medicine, London, UK, \\ 3 Computer Science department, University of Verona, Verona, Italy \\ 4 Radiology Department, Centre Hospitalier Universitaire Vaudois (CHUV), \\ Switzerland \\ 5 University College London Hospitals, London, UK \\ ${ }^{6}$ UCL Centre of Medical Imaging Computing, Department of Medical Physics, \\ London, UK \\ e.bonet-carne@ucl.ac.uk,
}

\begin{abstract}
The VERDICT (Vascular, Extracellular and Restricted Diffusion for Cytometry in Tumours) technique estimates non-invasively cancer microstructure features. The clinical application of VERDICT for prostate cancer requires constraining some of the models parameter. This work uses the Accelerated Microstructure Imaging via Convex Optimization (AMICO) formulation for VERDICT (VERDICT-AMICO), to investigate parameter estimation for prostate tissue, in an attempt to minimize the parameter constraints. We examine various dictionaries for VERDICT-AMICO enabling different levels of flexibility on the choice of parameter values. Depending on the stability of the fitting this procedure leads to the selection of a dictionary (or dictionaries) with the fewest number of model parameter constraints. Results show that with the current VERDICT imaging acquisition, the model can have an extra free parameter to fit, the extracellular diffusivity. In conclusion, the AMICO adaptation for VERDICT allowed testing of different values for the previously fixed model parameters, and helped relax assumptions of fixed extracellular diffusivity that the model currently uses for prostate cancer characterisation.
\end{abstract}

Keywords: VERDICT-AMICO, microstructure characterisation, prostate cancer

\section{Introduction}

Prostate cancer is the most common cancer among men in all economically developed countries[1]. A non-invasive method of diagnosis and grading would revolutionise clinical practice. The VERDICT (Vascular, Extracellular, and Restricted 
Diffusion for Cytometry in Tumours) model[2,3] is a three compartment modelbased technique that is designed to capture the main microstructural properties of cancerous tissue. VERDICT has shown promising results in preclinical studies for characterising microstructural tissue properties of xenograft tumour models [3] and in a pilot clinical setting for discriminating benign and malignant prostate tissue [2]. Currently, VERDICT is being tested as part of the INNOVATE clinical trial [4] for prostate cancer characterisation. Diffusion-weighted MRI (DW-MRI) is becoming increasingly important in the assessment of malignant tumours [5]. Model-based quantitative imaging techniques like VERDICT can ameliorate problems with simplistic diffusion-based indices, such as Apparent Diffusion Coefficient (ADC), by estimating parameters reflecting separate biophysical influences on the signal. However, the original implementation of VERDICT requires a computationally expensive non-linear fitting procedure to estimate the model parameters, which limits its use in large cohort studies and real-time clinical applications. Ultrafast fitting algorithms, such as the Accelerated Microstructure Imaging via Convex Optimization (AMICO) framework [6], have been developed to address the computational cost of model-based microstructure-imaging techniques.

The first implementation of the AMICO formulation for the VERDICT model (VERDICT-AMICO) in prostate tissue [7] performed a direct comparison of non-linear fitting and AMICO fitting for the VERDICT signal model and parameter constraints used in [2]. VERDICT-AMICO showed a good agreement with the non-linear VERDICT fitting. Parameter maps obtained revealed qualitatively similar differences between tumour and normal tissue. Furthermore, VERDICT-AMICO computes the estimates for tissue microstructure faster than the previous non-linear fitting technique and locates the global minimum parameter values more reliably. However, VERDICT-AMICO inherits some parameter constraints from the original non-linear implementation, which could be relaxed due to the enhanced stability of the AMICO fitting.

Here, we aim to find a stable implementation for the VERDICT model that imposes the least constraints on the parameters values. Thus, we use AMICO to fit the VERDICT model for prostate cancer as published previously [7] and to explore the possibility of unfixing previously fixed model parameters by trying various dictionaries for VERDICT-AMICO. Specifically, we investigate which parameters should remain fixed due to lack of sensitivity intrinsic in the data (i.e. number of acquisitions), and which constraints can be relaxed to increase biological realism. This improves the quantitative nature of the maps we obtain; we also consider retaining qualitative conspicuity for the original parametric maps in the cancer lesion areas, which are clinically important.

\section{Material and Methods}

This section describes the VERDICT model and VERDICT-AMICO [7], the direct adaptation of VERDICT model for prostate [2] using the AMICO framework [6]. We also provide details of the MRI acquisition and the patient population. 


\subsection{VERDICT}

The VERDICT model characterizes water diffusion in three different compartments in tumours: vascular (VASC), extracellular-extravascular (EES), and intracellular (IC). Mathematically, VERDICT is the addition of three parametric models without considering exchange between them. The normalized diffusion signal for the VERDICT model is:

$$
S=\sum_{i=1}^{3} f_{i} S_{i}
$$

where $f_{i}$ is the proportion of signal with no diffusion weighting $(\mathrm{b}=0)$ from water molecules in population $i$ (VASC, EES or IC), $0 \leq f_{i} \leq 1, \sum_{i=1}^{3} f_{i}=1$.

In the VERDICT model for prostate cancer in [2] the diffusion signal for the intracellular (IC) compartment is modelled with impermeable spheres and has (IC diffusivity) and (cell radius) as parameters. The model for the extracellularextravascular space (EES) compartment is an isotropic diffusion tensor with (diffusivity EES) as parameters. The vascular compartment uses the "Astrostick" model (using the terminology of [8] for isotropically distributed negligibly-thin restricting cylinders) and has (pseudo-diffusivity) as parameters. The clinical implementation of the VERDICT model has only 3 independent unfixed parameters: $f_{I C}$, $R$ and $f_{E E S}$. The vascular volume fraction was calculated as $f_{V A S C}=1-$ $f_{I C}-f_{E E S}$, and the diffusion and pseudo-diffusion coefficients were fixed to $d_{I C}=d_{E E S}=2 \times 10^{-9} \mathrm{~m}^{2} / \mathrm{s}, P=8 \times 10^{-9} \mathrm{~m}^{2} / \mathrm{s}[2]$.

\subsection{VERDICT-AMICO}

Accelerated Microstructure Imaging via Convex Optimization (AMICO) have been developed to address the computational cost of model-based microstructureimaging techniques [6]. AMICO linearises the fitting problem using a dictionary composed with synthetic signals simulating the number of measurements per voxel $\left(N_{d}\right)$.

In the first implementation of VERDICT-AMICO, the dictionary $\Phi=\left\{\phi_{i j}\right\} \in$ $\Re_{+}^{N_{d} x N_{k}}$ was partitioned in three sub-matrices corresponding to the three VERDICT compartments:

$$
\Phi=\left[\Phi^{r}\left|\Phi^{e}\right| \Phi^{v}\right]
$$

where $\Phi^{r} \in \Re^{N_{d} x N_{r}}, \Phi^{e} \in \Re^{N_{d} x N_{e}}$ and $\Phi^{v} \in \Re^{N_{d} x N_{v}}$ model the IC, EES and vascular contributions of the diffusion signal in the voxel with $N_{k}=N_{r}+N_{e}+N_{v}$ The regularization function used is the basic Tikhonov regularization with the same $\lambda$ value $(\lambda=0.001)$ used in [7] that was set empirically. The full dictionary consists of $N_{k}=19$ entries in total combining three sub-dictionaries as follows:

- Each column in $\Phi^{r} \in \Re^{N_{d} x N_{r}}$ corresponds to the signal attenuation of the water molecules restricted within spheres with a specific radius. $N_{r}=17$ 
different radii values linearly spaced from 0.01 to $15.1 \mu \mathrm{m}$ (both values included). The corresponding signal profiles are estimated according to the sphere model (using the name from [8]), assuming $d_{I C}=2 \times 10^{-9} \mathrm{~m}^{2} / \mathrm{s}$.

- A single compartment, $\Phi^{e} \in \Re^{N_{d} x N_{e}}$ where $N_{e}=1$ describes the EES with the same fixed value for the diffusion coefficient $d_{E E S}=2 x 10^{-9} \mathrm{~m}^{2} / \mathrm{s}$. Signal is estimated according to the ball model (terminology in [8]).

- A single compartment, $\Phi^{v} \in \Re^{N_{d} x N_{v}}$ where $N_{v}=1$ is considered to account for the vascular volume fraction. Signal is estimated according to the "Astrostick" model and pseudo-diffusivity (perfusion) is fixed $P=8 \times 10^{-9} \mathrm{~m}^{2} / \mathrm{s}$.

\subsection{Patients and Data Acquisition and Processing}

This study was performed with local ethics committee approval as part as the INNOVATE clinical trial [4]. Between April and June 2016, 6 men were prospectively recruited and provided informed written consent. The inclusion criteria for these patients were (1) suspected prostate cancer or (2) undergoing active surveillance for known prostate cancer. Exclusion criteria were: (1) previous hormonal, radiation therapy or surgical treatment for prostate cancer and (2) biopsy within 6 months prior to the scan.

All patients underwent a standard multi-parametric prostate (mp)-MRI [9], on a 3T scanner (Achieva, Philips Healthcare, NL) supplemented by VERDICT DW-MRI. VERDICT DW-MRI was acquired using a pulse-gradient spin-echo sequence and an optimized imaging protocol for prostate adapted from [10]with five $\mathrm{b}$ values between $90-3000 \mathrm{~s} / \mathrm{mm}$ in 3 orthogonal directions. Table 1 summarises the $\Delta, \delta,|G|$, TE and TR corresponding to each b-value. VERDICT-MRI data was registered using a rigid registration $[11,12]$ and then normalised with the $b=0$ image for every TE to avoid T2 dependence. The number of normalized measurements per voxel is $N_{d}=20$.

Table 1. Optimized imaging protocol for VERDICT prostate characterisation. $\Delta$ is the gradient separation time, $\delta$ is the gradient duration, $|G|$ is the gradient strength, $\mathrm{TE}$ is the echo time and TR is the repetition time.

\begin{tabular}{llllll}
\hline \hline b-values $[s / m m]$ & $\Delta[m s]$ & $\delta[m s]$ & $|G|[m s]$ & $T E[m s]$ & $T R[m s]$ \\
\hline 90 & 23.8 & 3.9 & 61.2 & 50 & 2482 \\
500 & 31.3 & 11.4 & 44.3 & 65 & 2482 \\
1500 & 43.8 & 23.9 & 32.0 & 90 & 2482 \\
2000 & 34.3 & 14.4 & 67.7 & 71 & 3945 \\
3000 & 38.8 & 18.9 & 60.0 & 80 & 3945 \\
\hline
\end{tabular}

MR datasets were visualized with Osirix Version 7.0 (Bernex, Switzerland). For each subject a board-certified radiologist (EJ) manually contoured (Osirix 7.0) different regions of interest (ROIs) in the VERDICT-AMICO maps corresponding to tumour and normal tissues in transition zone (TZ) and peripheral 
zone (PZ). The total number of ROIs was 20. To examine similar histological areas, we grouped the ROIs according to tumour grading and the prostate zone, which resulted in 10 different ROI groups (g-ROI) with homogeneous tissue.

\section{Experiments and Results - Parameter Fitting}

The first experiments (3.1) explore the free VERDICT model using AMICO with a wide range of parameters values. Subsequent experiments (3.2-3.3) explore which parameters can be fixed to ensure fitting stability, while retaining an extensive dictionary for the unfixed parameters. The different dictionaries used are summarised in Table 2.

\subsection{Unfixed model}

The VERDICT model fitting in prostate requires fixed parameters to achieve stable estimates [2]. This experiment uses the free tissue model to investigate the range of the parameter estimates with VERDICT-AMICO. The number of measurements limits the dictionary size for the VERDICT-AMICO implementation and this restricts the values for the selected ranges in each sub-matrix. Accordingly, we use four different dictionaries for fixing different pseudo-diffusivity $P$ values in each while unfixing $d_{I C}$ and $d_{E E S}$. We retain the following constraints for physical realism: $1 \times 10^{-11} \mathrm{~m}^{2} / \mathrm{s} \leq d_{I C} \leq 2.9 \times 10^{-9} \mathrm{~m}^{2} / \mathrm{s}, d_{I C}=d_{E E S}$ and $3 \times 10^{-9} \mathrm{~m}^{2} / \mathrm{s} \leq P \leq 9 \times 10^{-8} \mathrm{~m}^{2} / \mathrm{s}$. The full dictionaries for VERDICT-AMICO consist of $N_{k}=19$ entries, as follows:

- $N_{r}=15$ combinations of 5 different radii (linearly spaced from 0.01 to $15.1 \mu \mathrm{m})$ and 3 different $d_{I C}\left(1.2 \times 10^{-9}, 1.7 \times 10^{-9}\right.$ and $\left.2.1 \times 10^{-9} \mathrm{~m}^{2} / \mathrm{s}\right)$.

- $N_{e}=3$ describes the EES with the same fixed value for the diffusion coefficient $d_{E E S}=1.2 \times 10^{-9}, 1.7 \times 10^{-9}$ and $2.1 \times 10^{-9} \mathrm{~m}^{2} / \mathrm{s}$ ).

- $N_{v}=1$, was fixed for each dictionary. The four different values are: (1) $P=3 \times 10^{-9} \mathrm{~m}^{2} / \mathrm{s},(2) P=1 \times 10^{-8} \mathrm{~m}^{2} / \mathrm{s},(3) P=3 \times 10^{-8} \mathrm{~m}^{2} / \mathrm{s}$ and $(4) P=$ $6 x 10^{-8} \mathrm{~m}^{2} / \mathrm{s}$. Signal is estimated according to the "Astrostick" model in [8].

Fig. 1 shows that VERDICT-AMICO performance for the new dictionaries (with unfixed $d_{I C}$ and $d_{E E S}$ parameters) is unstable for most parameters and especially for $R, f_{V A S C}$ and $f_{E E S}$ where the estimates present high variations depending on the dictionary. The parameter values obtained with the original dictionary (fixed values for $P, d_{I C}$ and $d_{E E S}$ ) are also shown as reference. Results also reveal a trend (values are not fluctuating randomly) in the values of the perfusion coefficient $P$ (or pseudo-diffusivity), allowing us to identify a value and fix this parameter.

\subsection{Fixing Parameters - Perfusion}

Since values show little variation, here we examine different pairings of and to find at which value the should be fixed, as different $d_{I C}$ values had only a minor 
Table 2. Summary of the dictionaries to show which parameter is fixed and its value. Values in bold are unfixed. $d_{I C}$ and $d_{E E S}$ values are multiplied by $10^{-9}$ if not stated differently.

\begin{tabular}{|c|c|c|c|c|c|c|c|}
\hline Model/Experiment & $f_{I C}$ & $\begin{array}{l}d_{I C} \\
{\left[m^{2} / s\right]}\end{array}$ & $R[\mu m]$ & $f_{E E S}$ & $\begin{array}{l}d_{E E S} \\
{\left[\mathrm{~m}^{2} / \mathrm{s}\right]}\end{array}$ & $f_{V A S C}$ & $\begin{array}{l}P \\
{\left[m^{2} / s\right]}\end{array}$ \\
\hline VERDICT[2] & $0-1$ & 2 & 0.01-15 & $0-1$ & 2 & $\begin{array}{l}1-f_{I C} \\
-f_{E E S} \\
\end{array}$ & $8 x 10^{-9}$ \\
\hline $\begin{array}{l}\text { VERDICT- } \\
\text { AMICO }[7]\end{array}$ & 0-1 & 2 & $0.01-15.1$ & $0-1$ & 2 & $0-1$ & $8 x 10^{-9}$ \\
\hline $\begin{array}{l}\text { Experiment } 3.1 \\
\text { (unfixed models) }\end{array}$ & 0-1 & $1.2-2.1$ & 0.01-15.1 & $0-1$ & $1.2-2.1$ & $0-1$ & $\begin{array}{l}3 \times 10^{-9} \\
1 \times 10^{-8} \\
3 x 10^{-8} \\
6 x 10^{-8}\end{array}$ \\
\hline $\begin{array}{l}\text { Experiment } 3.2 \\
\text { (fixed } d_{I C}-P \text { pairs) }\end{array}$ & 0-1 & $\begin{array}{l}\{1.0, \quad 1.2, \\
1.5, \quad 1.8, \\
2.0, \quad 2.1 \\
2.3, \quad 2.6 \\
2.8,3.1\}\end{array}$ & 0.01-15.1 & 0-1 & $1.1-3.1$ & 0-1 & $\begin{array}{l}\left\{8 x 10^{-9}\right. \\
1 x^{10-8} \\
3 x 10^{-8} \\
6 x 10^{-8} \\
\left.1 x 10^{-7}\right\}\end{array}$ \\
\hline Experiment 3.3 & $0-1$ & $\begin{array}{lr}\left\{8 x 10^{-10},\right. \\
1.0, & 1.2, \\
1.4, & 1.5, \\
1.6, & 1.8, \\
2.0, & 2.1 \\
2.2, & 2.3, \\
2.6, & 2.8, \\
2.9, & 3.1\}\end{array}$ & 0.01-15.1 & 0-1 & 1.1-3.1 & $0-1$ & $8 x 10^{-9}$ \\
\hline $\begin{array}{l}\text { VERDICT-AMICO } \\
\left.\text { (unfixed } d_{E E S}\right)\end{array}$ & 0-1 & 2 & $0.01-15.1$ & $0-1$ & 1.1-3.1 & 0-1 & $8 x 10^{-9}$ \\
\hline
\end{tabular}



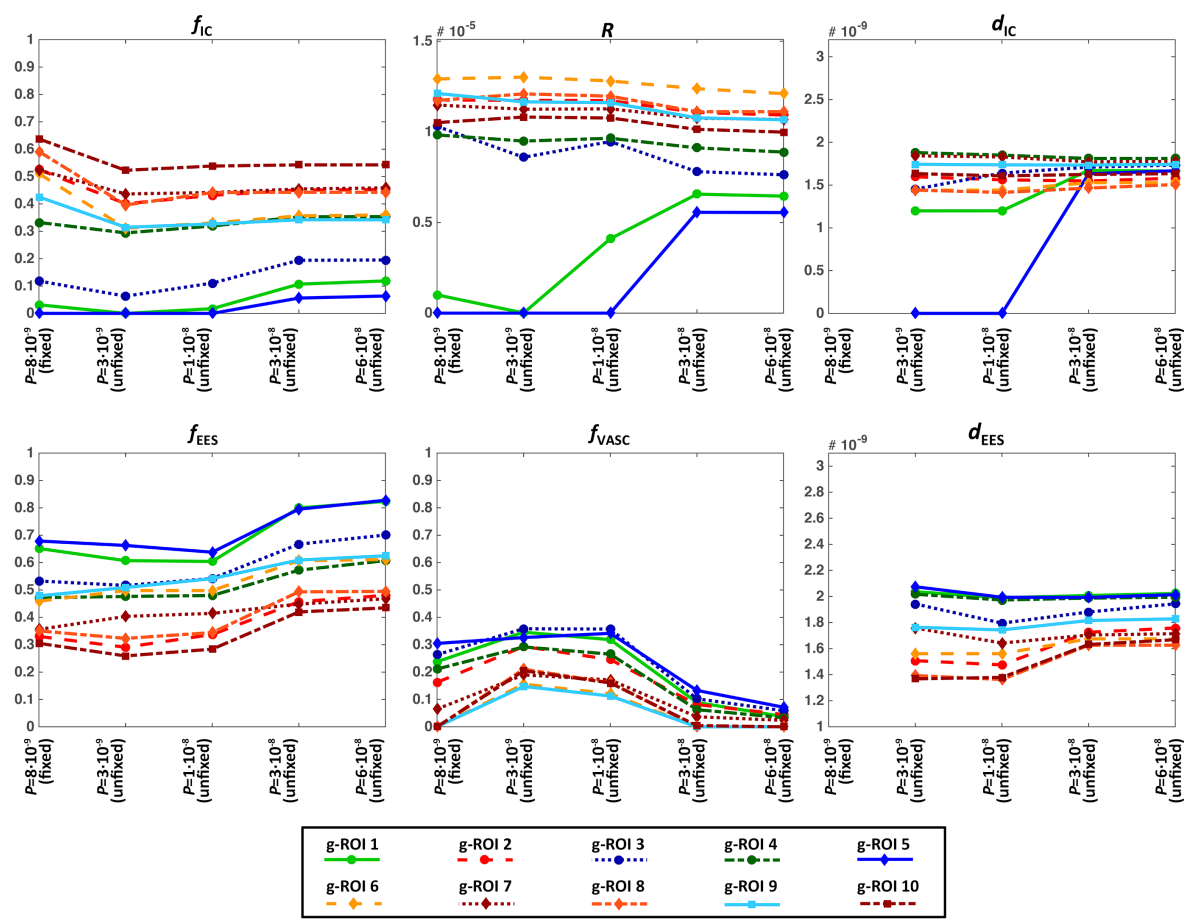

Fig. 1. Parameter estimates for all voxels within each of the grouped-ROIs (g-ROI), median values represented. First measures correspond to $P=8 \times 10^{--9} \mathrm{~m}^{2} / \mathrm{s}$ with fixed values for $d_{I C}$ and $d_{E E S}$. The other points correspond to estimated values using the dictionary with unfixed $d_{I C}$ and $d_{E E S}$ for different fixed perfusion values. $R$ in $[\mu \mathrm{m}]$. 
effect on the fitting. This is also supported by [13], where the study finds minimal effects from fixing the intracellular diffusivity.

In this work, fixing $d_{I C}$ and $P$ allows us (i) to increase the range of radii values in the dictionary, which could potentially enable capturing a greater range of cell types with different cell sizes, and (ii) to increment the range of $d_{E E S}$ values, which could be useful for detecting different EES tissue features (e.g. to better characterize lumen and large stromal cells).

To fix $P$ we first test 50 different $d_{I C}-P$ fixed pairs using the following dictionary for the rest of the parameters:

- $N_{r}=13$ different radii (linearly spaced from 0.01 to $15.1 \mu \mathrm{m}$ ).

- $N_{e}=5$ diffusion coefficient for EES. $d_{E E S}=1.1 \times 10^{-9}, 1.6 \times 10^{-9}, 2.1 \times 10^{-9}$, $2.6 \times 10^{-9}$ and $3.1 \times 10^{-9} \mathrm{~m}^{2} / \mathrm{s}$ ).

- $N_{v}=1$, fixed for each dictionary.

The fixed values for the $d_{I C}-P$ combinations are:

- 5 values for perfusion: $P=8 x 10^{-9} \mathrm{~m}^{2} / \mathrm{s}, P=1 \times 10^{-8} \mathrm{~m}^{2} / \mathrm{s}, P=3 x 10^{-8} \mathrm{~m}^{2} / \mathrm{s}$, $P=6 \times 10^{-8} \mathrm{~m}^{2} / \mathrm{s}$ and $P=1 \times 10^{-7} \mathrm{~m}^{2} / \mathrm{s}$.

- 10 values for intracellular diffusivity: $d_{I C}=1.0 \times 10^{-9}, 1.2 \times 10^{-9}, 1.5 \times 10^{-9}$, $1.8 \times 10^{-9}, 2.0 \times 10^{-9}, 2.1 \times 10^{-9}, 2.3 \times 10^{-9}, 2.6 \times 10^{-9}, 2.8 \times 10^{-9}$ and $\left.3.1 \times 10^{-9} \mathrm{~m}^{2} / \mathrm{s}\right)$.

We compute the median value for each parameter for all voxels within each of the grouped-ROIs for each $d_{I C}-P$ pair. Most of the estimates plateau or present minor variations for all the values (with fixed intracellular diffusivity) for the majority of both ROIs and g-ROIs. This trend can be qualitatively observed in the $f_{I C}$ maps for two different patients in Fig.2.

The radiologist EJ examined the VERDICT maps for tumour conspicuity to ensure tumour enhancement and clinical relevance. In general, all maps appear similar for different perfusion coefficients with small variations revealed by the objective function. As in previous results (see Fig. 1) the perfusion value used for the dictionary has minor effect on the fitting. Thus, we fix perfusion to the original value $P=8 \times 10^{-9} \mathrm{~m}^{2} / \mathrm{s}$ without compromising qualitative tumour conspicuity.

\subsection{Fixing Parameters - Intracellular Diffusivity}

To identify the best value to fix the intracellular diffusivity parameter we fix perfusion at the original value and repeat the same experiment increasing the number of values for $d_{I C}$. We test 15 values for intracellular diffusivity: $d_{I C}=$ $8.0 \times 10^{-10}, 1.0 \times 10^{-9}, 1.2 \times 10^{-9}, 1.4 \times 10^{-9}, 1.5 \times 10^{-9}, 1.6 \times 10^{-9}, 1.8 \times 10^{-9}, 2.0 \times 10^{-9}$, $2.1 \times 10^{-9}, 2.2 \times 10^{-9}, 2.3 \times 10^{-9}, 2.6 \times 10^{-9}, 2.8 \times 10^{-9}, 2.9 \times 10^{-9}$ and $3.1 \times 10^{-9} \mathrm{~m}^{2} / \mathrm{s}$.

Fig. 3 shows the median value for each $d_{I C}$ value for the g-ROIs, each represented in different colours. Most of the estimates, including the objective function, present small variations within the varying intracellular diffusivity as in [13], so we fix the diffusivity at $d_{I C}=2.0 \times 10^{-9} \mathrm{~m}^{2} / \mathrm{s}$, which also corresponds to the original fixed value in [2]. Hence, the final VERDICT-AMICO dictionary with an extra un-fixed parameter $\left(d_{E E S}\right)$ is: 


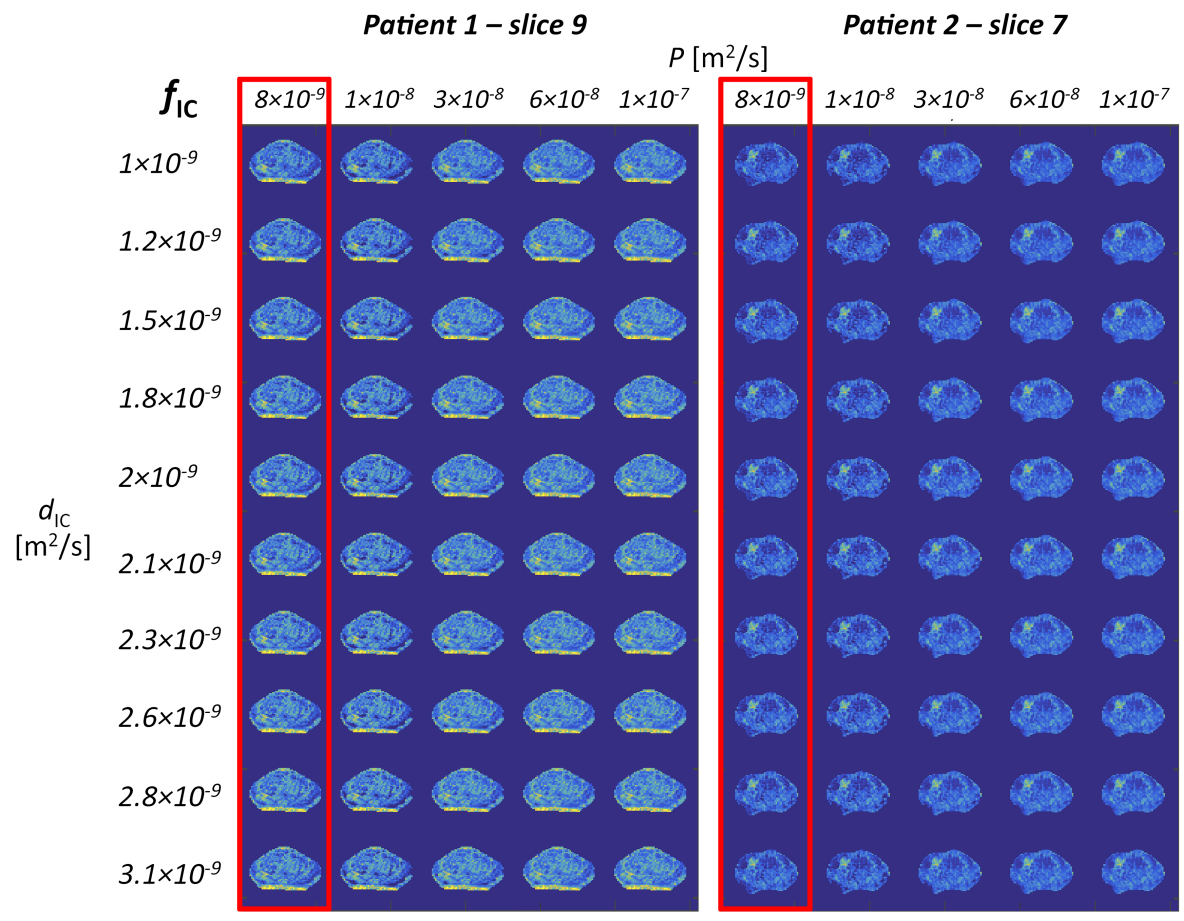

Fig. 2. Qualitative evaluation of VERDICT-AMICO for various fixed $d_{I C}-P$ pairs in two different patients. $f_{I C}$ parametric maps for each $d_{I C}-P$ pair for two different slices from two patients. Each row has the same fixed $d_{I C}$ while each column has a fixed $P$. The red rectangles indicate which maps correspond to the selected fixed perfusion for different $d_{I C}$. 
- $N_{r}=13$ different radii (linearly spaced from 0.01 to $15.1 \mu \mathrm{m}$ ) with fixed $d_{I C}=2.0 \times 10^{-9} \mathrm{~m}^{2} / \mathrm{s}$.

- $N_{e}=5$ diffusion coefficient for EES: $d_{E E S}=1.1 \times 10^{-9}, 1.6 \times 10^{-9}, 2.1 \times 10^{-9}$, $2.6 \times 10^{-9}$ and $3.1 \times 10^{-9} \mathrm{~m}^{2} / \mathrm{s}$ ).

- $N_{v}=1$, with $P=8.0 \times 10^{-9} \mathrm{~m}^{2} / \mathrm{s}$.
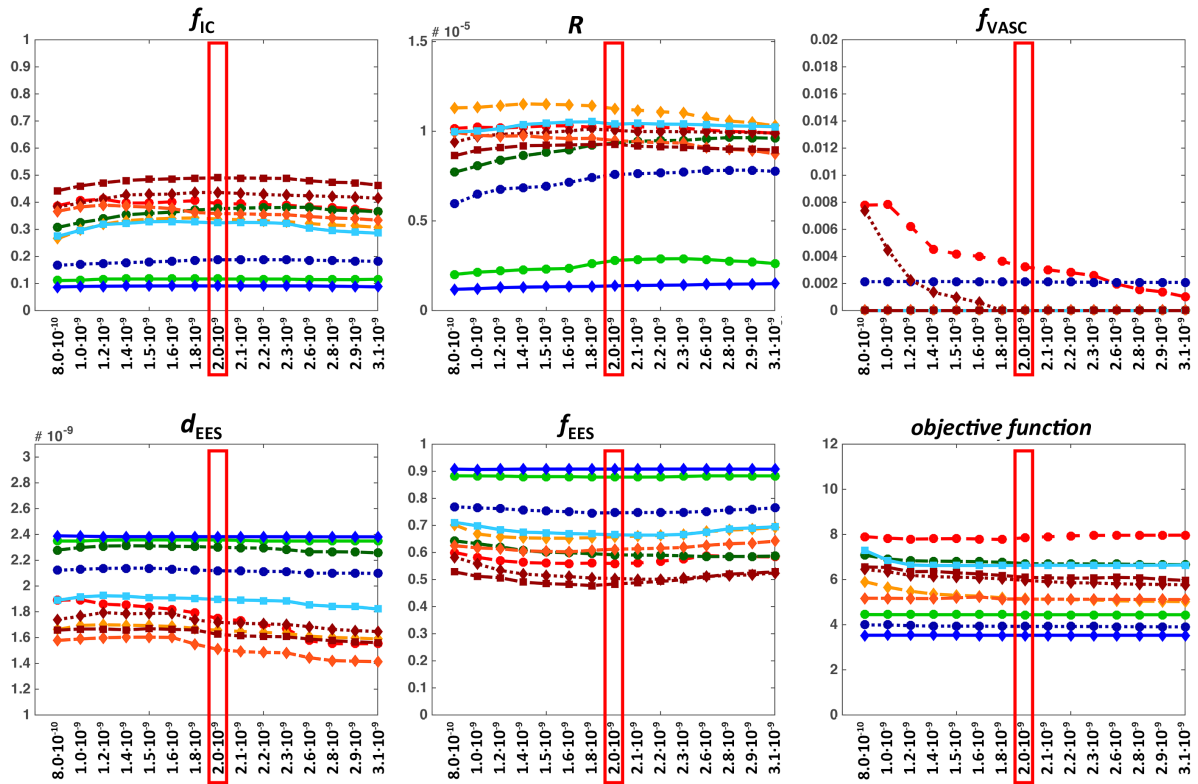

$f_{\mathrm{EES}}$

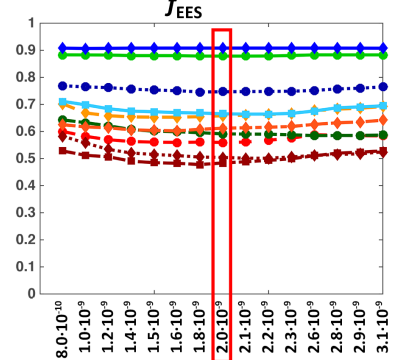

objective function

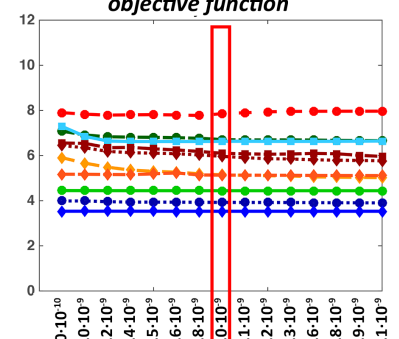

\begin{tabular}{|c|c|c|c|c|}
\hline g-ROI 1 & g-ROI 2 & g-ROI 3 & g-ROI 4 & $\stackrel{\text { g-ROI } 5}{\longrightarrow}$ \\
\hline g-ROI 6 & $\begin{array}{c}\text { g-ROI } 7 \\
\cdots+\cdots\end{array}$ & $\begin{array}{l}\text { g-ROI } 8 \\
-=-\leftarrow=-\end{array}$ & $\mathrm{g}-\mathrm{ROI} 9$ & g-ROI 10 \\
\hline
\end{tabular}

Fig. 3. Parameter performance when changing $d_{I C}$ for fixed $P=8.0 x 10^{-9} \mathrm{~m}^{2} / \mathrm{s}$. The median value for each parameter for all voxels within each of the g-ROIs (represented in different lines) for each $d_{I C}$ value (horizontal axis correspond to the 15 different values for $\left.d_{I C}\right)$.

\subsection{New Parameter estimation}

Fig. 4 shows a qualitative comparison for two different patients for the additional maps from VERDICT-AMICO, the $\left(d_{E E S}\right)$ map and an example of a parametric 
combination $\left(f_{I C} / d_{E E S}\right)$ that increases tumour conspicuity. A standard T2 image part of the mp-MRI evaluation with the ROIs is also shown for anatomical comparison purposes.

\section{Patient 1 - slice 9}
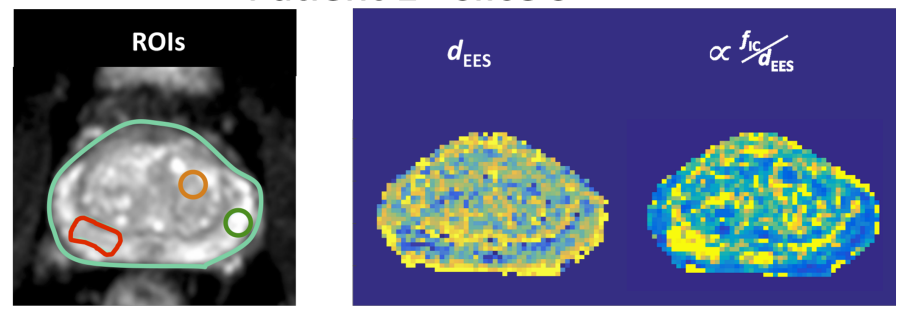

$\boldsymbol{d}_{\mathrm{EES}}$
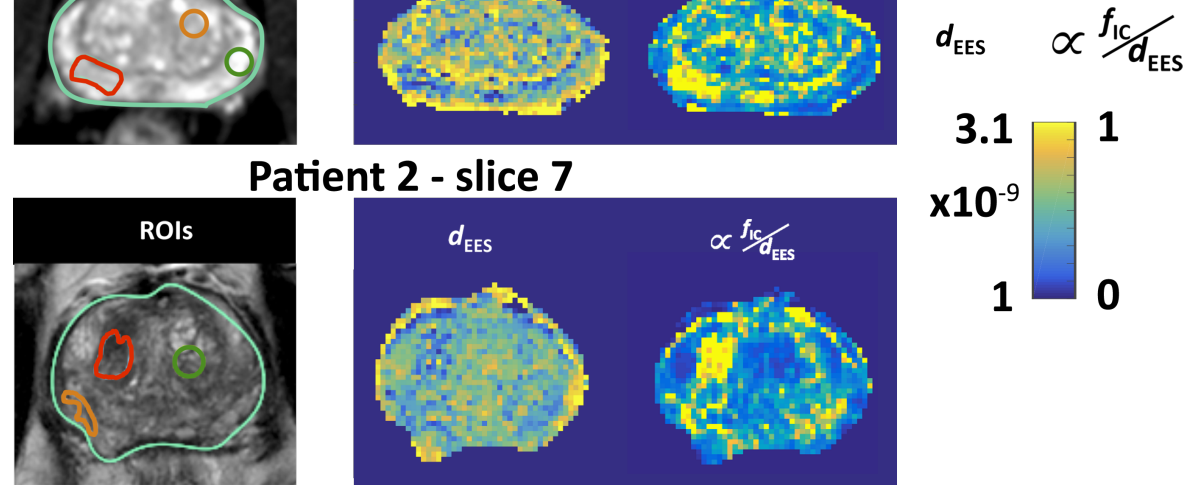

Patient 2 - slice 7
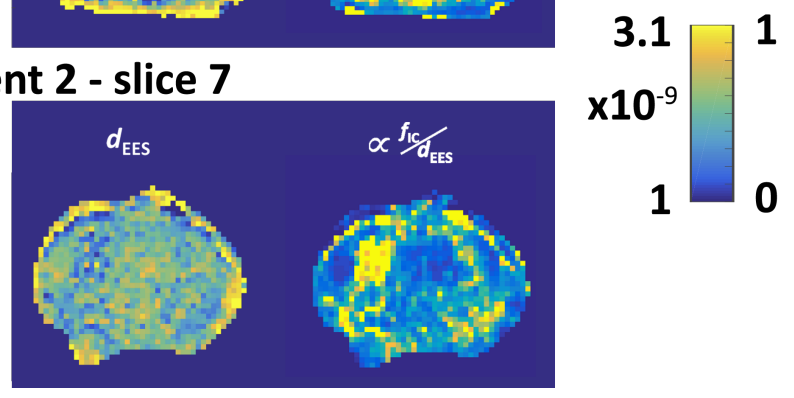

Fig. 4. Qualitative Comparison of new VERDICT-AMICO maps for two different patients. ROIs in T2 image: the whole prostate (light-green-bigger ones). ROI description from left to right: Patient 1, Tumour-PZ (red), suspected-TZ (orange) and Benign-PZ (green). Patient 2, suspected-PZ (orange), Tumour-TZ (red) and Benign-PZ (green).

Fig. 5 illustrates the parameter estimates for all voxels within four of the g-ROIs, normal and Gleason Score $3+4$ in $\mathrm{TZ}$ and PZ. The new values for the AMICO-VERDICT dictionary compute similar estimates compared with the original fitting for $f_{I C}, f_{E E S}$ and $R$. We note that the vascular volume fraction $f_{V A S C}$ is lower for most g-ROIs, showing that unfixing the $d_{E E S}$ one of the limitations of VERDICT could be solved. These results are also similar for the individual ROI analysis (not shown).

\section{Discussion}

We use VERDICT-AMICO to relax the original VERDICT model constraints for prostate cancer characterisation. The improvement in calculation speed that 

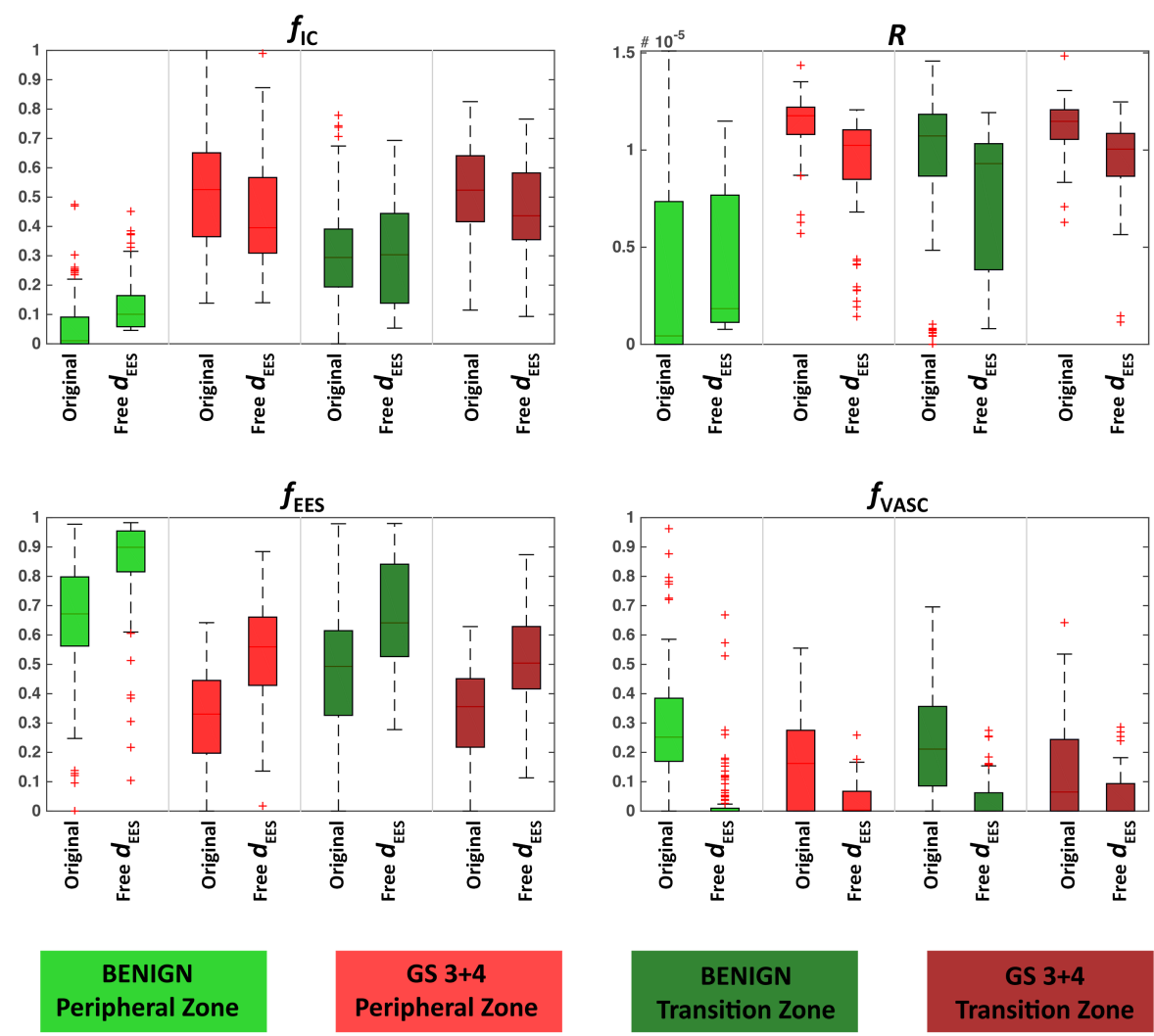

Fig. 5. Parameter estimates comparison for all voxels within four of the g-ROIs using the original VERDICT-AMICO dictionary and the one with unfixed $d_{E E S}$. 
AMICO framework offers makes it practical to try different dictionaries for the identification of potentially stable parameter combinations and to help reduce assumptions of fixed compartmental diffusivities that VERDICT studies currently use. We search for dictionary that allows us to reduce the VERDICT parameter constraints and formulate a new version of the model for prostate cancer. Results show that it is possible to eliminate one of the original parameter constraints and unfix the previously fixed $d_{E E S}$ parameter allowing for a new diffusivity map.

First we tested the AMICO implementation using the VERDICT prostate model with all the model parameters unfixed. However, this version was unstable as the signal cannot support unambiguous estimation of so many parameters, so we searched for the best constraints to impose. We fix the perfusion parameter to the original value of $P=8 \times 10^{-9} \mathrm{~m}^{2} / \mathrm{s}$ and tested the rest of the parameters with a wide range of fixed $d_{I C}$ values to find the best $d_{I C}$ coefficient. We confirmed that different $d_{I C}$ values have small impact on the other parameter estimates with fixed perfusion, as previous studies [13]. Thus, we fixed $d_{I C}$ to the original value for this parameter $d_{I C}=2 \times 10^{-9} \mathrm{~m}^{2} / \mathrm{s}$ [2], and obtained the final dictionary. The parameter estimates from the new dictionary are more biophysical plausible and in close agreement with histology (i.e. $f_{V A S C}$ ). Also, the estimated values for $f_{I C}, R$ or $f_{E E S}$ do not hit the boundary constraints after unfixing $d_{E E S}$, which is evidence of improved and stable fitting. To gain sufficient sensitivity for allowing estimation of the full model without constraints would require a much wider sampling of the range of b-values and diffusion times, which is not clinically practical with the existing technology. Currently, the VERDICT DW-MRI acquisition is less than 15 minutes, which is within the time constraints to be used in clinical practice.

The main limitation of the AMICO framework is that the fitting results depend to some extent on the dictionary values, as the regularization has to be set empirically. Graphical Processor Units (GPUs) could also be used to optimise MRI models non-linearly [14] and avoid the dictionary dependency. We used histological information to guide us in the selection of values for our dictionary. However, more samples from larger datasets are required to validate the VERDICT parameters with histology. Finally, the inherent limitations of model-based approaches have to be taken into consideration when interpreting the estimated values. Nevertheless, the results from this study and previous work provide good evidence that this form of the VERDICT prostate model can provide useful information, improving current methods.

To conclude, the AMICO adaptation for VERDICT allowed testing of different values for the previously fixed model parameters in a practical time and helped relax assumptions of fixed compartmental diffusivities that VERDICT currently uses for prostate cancer characterisation.

\section{References}

1. Torre, L.A., Bray, F., Siegel, R.L., Ferlay, J., Lortet-Tieulent, J., Jemal, A.: Global Cancer Statistics, 2012 
2. Panagiotaki, E., Chan, R.W., Dikaios, N., Ahmed, H., O'Callaghan, J., Freeman, A., Atkinson, D., Punwani, S., Hawkes, D.J., Alexander, D.C.: Microstructural Characterization of Normal and Malignant Human Prostate Tissue With Vascular, Extracellular, and Restricted Diffusion for Cytometry in Tumours Magnetic Resonance Imaging. Invest. Radiol. 50(4) (2015) 218-227

3. Panagiotaki, E., Walker-Samuel, S., Siow, B., Johnson, S.P., Rajkumar, V., Pedley, R.B., Lythgoe, M.F., Alexander, D.C.: Noninvasive quantification of solid tumor microstructure using VERDICT MRI. Cancer Res. 74(7) (apr 2014) 1902-12

4. Johnston, E., Pye, H., Bonet-Carne, E., Panagiotaki, E., Patel, D., Galazi, M., Heavey, S., Carmona, L., Freeman, A., Trevisan, G., Allen, C., Kirkham, A., Burling, K., Stevens, N., Hawkes, D., Emberton, M., Moore, C., Ahmed, H.U., Atkinson, D., Rodriguez-Justo, M., Ng, T., Alexander, D., Whitaker, H., Punwani, S.: INNOVATE: A prospective cohort study combining serum and urinary biomarkers with novel diffusion-weighted magnetic resonance imaging for the prediction and characterization of prostate cancer. BMC Cancer 16(1) (dec 2016) 816

5. Padhani, A.R., Liu, G., Koh, D.M., Chenevert, T.L., Thoeny, H.C., Takahara, T., Dzik-Jurasz, A., Ross, B.D., Van Cauteren, M., Collins, D., Hammoud, D.A., Rustin, G.J.S., Taouli, B., Choyke, P.L.: Diffusion-weighted magnetic resonance imaging as a cancer biomarker: consensus and recommendations. Neoplasia 11(2) (mar 2009) 102-25

6. Daducci, A., Canales-Rodríguez, E.J., Zhang, H., Dyrby, T.B., Alexander, D.C., Thiran, J.P.: Accelerated Microstructure Imaging via Convex Optimization (AMICO) from diffusion MRI data. Neuroimage 105 (jan 2015) 32-44

7. Bonet-Carne, E., Daducci, A., Panagiotaki, E., Johnston, E., Stevens, N., Atkinson, D., Shonit Punwani, S., Alexander, D.: Non-invasive quantification of prostate cancer using AMICO framework for VERDICT MR. In: Int. Soc. Magn. Reson. Med. (2016)

8. Panagiotaki, E., Schneider, T., Siow, B., Hall, M.G., Lythgoe, M.F., Alexander, D.C.: Compartment models of the diffusion MR signal in brain white matter: a taxonomy and comparison. Neuroimage 59(3) (feb 2012) 2241-54

9. Barentsz, J.O., Richenberg, J., Clements, R., Choyke, P., Verma, S., Villeirs, G., Rouviere, O., Logager, V., Fütterer, J.J.: ESUR prostate MR guidelines 2012. Eur. Radiol. 22(4) (2012) 746-757

10. Panagiotaki, E., Ianus, A., Johnston, E., Chan, R., Atkinson, D., Punwani, S., Hawkes, D., Alexander, D.: Optimised VERDICT MRI protocol for prostate cancer characterisation. In: Proc. 23rd Meet. Int. Soc. Magn. Reson. Med. 2015. 2872

11. Ourselin, S., Roche, A., Subsol, G., Pennec, X., Ayache, N.: Reconstructing a 3D structure from serial histological sections. Image Vis. Comput. 19(1-2) (2001) 25-31

12. Ourselin, S., Stefanescu, R., Pennec, X. In: Robust Registration of Multi-modal Images: Towards Real-Time Clinical Applications. Springer Berlin Heidelberg, Berlin, Heidelberg (2002) 140-147

13. Li, H., Jiang, X., Xie, J., Gore, J.C., Xu, J.: Impact of transcytolemmal water exchange on estimates of tissue microstructural properties derived from diffusion MRI (2016)

14. Harms, R., Fritz, F., Tobisch, A., Goebel, R., Roebroeck, A.: Robust and fast nonlinear optimization of diffusion MRI microstructure models. Neuroimage $\mathbf{1 5 5}$ (jul 2017) 82-96 